\title{
Review Article \\ Recent Advancements in Prognostic Factors of Epithelial Ovarian Carcinoma
}

\author{
Mohammad Ezzati, ${ }^{1,2}$ Amer Abdullah, ${ }^{3}$ Ahmad Shariftabrizi, ${ }^{4}$ June Hou, ${ }^{5}$ Michael Kopf, \\ Jennifer K. Stedman, ${ }^{3}$ Robert Samuelson, ${ }^{3}$ and Shohreh Shahabi ${ }^{3}$ \\ ${ }^{1}$ Department of Obstetrics and Gynecology, Washington Hospital Center, 110 Irving Street NW, Washington, DC 20010, USA \\ ${ }^{2}$ Department of Obstetrics and Gynecology, The University of Texas Southwestern Medical Center, \\ 5323 Harry Hines Boulevard, Dallas, TX 75390, USA \\ ${ }^{3}$ Department of Obstetrics, Gynecology and Reproductive Biology, Danbury Hospital, 24 Hospital Avenue, Danbury, CT 06810, USA \\ ${ }^{4}$ Department of Pathology and Laboratory Medicine, School of Medicine, Tufts University, \\ 800 Washington Street, Boston, MA 02111, USA \\ ${ }^{5}$ Division of Gynecologic Oncology, Department of Obstetrics and Gynecology and Women's Health, Albert Einstein College of \\ Medicine and Montefiore Medical Center, Montefiore Centennial, 3332 Rochambeau Avenue, Bronx, NY 10467-2836, USA \\ ${ }^{6}$ Department of Medicine, Danbury Hospital, 24 Hospital Avenue, Danbury, CT 06810, USA
}

Correspondence should be addressed to Shohreh Shahabi; shohreh.shahabi@wchn.org

Received 13 June 2014; Accepted 26 August 2014; Published 29 October 2014

Academic Editor: Yinhua Yu

Copyright ( 2014 Mohammad Ezzati et al. This is an open access article distributed under the Creative Commons Attribution License, which permits unrestricted use, distribution, and reproduction in any medium, provided the original work is properly cited.

Ovarian cancer remains the most common cause of gynecologic cancer-related death among women in developed countries. Nevertheless, subgroups of ovarian cancer patients experience relatively longer survival. Efforts to identify prognostic factors that characterize such patients are ongoing, with investigational areas including tumor characteristics, surgical management, inheritance patterns, immunologic factors, and genomic patterns. This review discusses various demographic, clinical, and molecular factors implicating longevity and ovarian cancer survival. Continued efforts at identifying these prognosticators may result in invaluable adjuncts to the treatment of ovarian cancer, with the ultimate goal of advancing patient care.

\section{Introduction}

There has been significant progress in the management of ovarian cancer, yet the disease still has the highest mortality of all pelvic malignancies combined. In the United States, in 2013, approximately 22,000 new cases of ovarian cancer will be diagnosed and over 14,000 women will die due to the disease [1]. Primary reasons for this poor prognosis include late stage of presentation due to the absence of symptoms, lack of effective screening tools, and the development of recurrent disease that is resistant to chemotherapy. The most common ovarian cancer is the epithelial subtype, of which over $70 \%$ of patients are presented with the advanced-stage disease, where the long term survival rate (10 years) is estimated at $15-30 \%$; in contrast the survival rate for early stage disease exceeds $90 \%$ [2-5].

The disparity in prognosis is largely a function of disease stage, which is the most important prognostic variable for ovarian cancer survival. However, a subgroup of patients is seemingly cured after standard therapy. Studies have also concentrated on the prognostic importance of tumor characteristics, surgical conditions, and demographic factors. More recently, attention has focused on the significance of molecular features, which are considered a reflection of tumor biology and might thus be utilized to predict response to various therapeutic modalities and determine the long term survival. 


\section{Age}

Age is a widely accepted prognostic factor of ovarian cancer $[6,7]$. Generally, a younger age at diagnosis portends a better prognosis, with women younger than 65 years having at least 2 years longer median survival compared to women older than 65 years; older women also have an increased risk of recurrence and death $[8,9]$. Age remains an independent prognostic factor after controlling for common confounding factors, such as performance status and medical comorbidities. Younger-aged women tend to have less invasive and well differentiated cancers and fewer comorbidities compared to older counterparts, yielding a more favorable overall prognosis $[10,11]$.

\section{Performance Status}

The Gynecologic Oncology Group adopted the Eastern Cooperative Oncology Group/World Health Organization scoring criteria for quantitative assessment of a patient's general health at the time of cancer diagnosis and prior to the start of treatment. Based on this system, the following performance status scores are given [12]: 0 is asymptomatic; 1 is symptomatic but completely ambulatory; 2 is symptomatic, $<50 \%$ in bed during the day; 3 is symptomatic, $>50 \%$ in bed, but not bedbound; 4 is completely bedbound.

A better performance status generally confers a greater tolerance to various therapeutic modalities, from surgery to chemotherapy, and perhaps motivates the adoption of a more aggressive treatment plan by clinicians. Several studies support this rationale and confirm the independent prognostic significance of performance status [9, 13-15].

\section{Tumor Stage and Ascites}

Staging systems describe the severity of a patient's cancer in a terminology that is shared across medical disciplines. While staging was originally developed to optimize treatment planning, a lower stage at diagnosis generally translates into a superior clinical outcome and improved survival $[5,16]$. The International Federation of Gynecology and Obstetrics (FIGO) developed the ovarian cancer staging system. This system emphasizes surgical evidence and correlates with the widely used TNM classification system. The FIGO system has been extensively studied over the past two decades and its independent prognostic significance is well established $[13,17-19]$. Patients with stage I disease largely enjoy a greater than 95\% 5-year survival, versus a less than 10\% 5-year survival of patients with stage IV disease [20-22].

From a clinical perspective, a higher stage signifies more extensive disease that is less likely to be optimally debulked, compared to a tumor that is confined to the pelvis. The radical surgeries needed to achieve optimal surgical debulking in extra-pelvic (stage III) and extra-abdominal (stage IV) disease also correlate with a higher incidence of perioperative morbidities. Additionally, the presence of a malignant pleural effusion, in the absence of any other criteria for stage IV disease, indicates a significantly poorer prognosis compared to stage III disease [23]; this finding further emphasizes the prognostic significance of the FIGO staging system. A biological explanation for this significance may lie in the possibility that a higher stage reflects a change in tumor biology, to a more aggressive state [23].

Ascites distinguishes a poorer prognosis in early-stage disease, contributes to the morbidity associated with advanced disease and is thus an important component of the FIGO staging system. Stage I and II diseases are upstaged to subclass $\mathrm{C}$ when there are malignant cells detected in ascitic fluid. In terms of management, patients with early-stage disease and ascites cannot be surgically cured. Ascites is thus considered a marker of unfavorable prognosis in earlystage disease and studies suggest that these patients should receive adjuvant chemotherapy following surgical debulking. However, optimal treatment for early-stage disease remains to be determined [24-27]. Considering the aforementioned prognostic significance of the FIGO staging system, it follows that several studies report an independent prognostic significance for the presence or absence of ascites in advanced-stage disease [17, 28-30]. The significance of ascites in advanced-stage disease is further recognized by its incorporation into a nomogram validated to predict survival in epithelial ovarian cancer $[31,32]$.

\section{Tumor Grade}

The degree of cellular differentiation, or tumor grade, has long been considered a factor influencing tumor behavior and affecting survival. This understanding derives from the fact that poorly differentiated cells represent a higher degree of aberrant mitoses, which equates with a more aggressive cancer cell behavior. However, studies conflict over the association between tumor grade and clinical outcome [9, $11,13,17,29,33,34]$. Part of the controversy results from the absence of a universally accepted grading system [35, 36]. A majority of pathologists employ a system introduced by FIGO in the 1970s that uses cellular architecture as the defining characteristic of tumor grade [37]. A subsequently developed system incorporates cellular architecture, nuclear grade, and mitotic activity [38, 39]; this system correlates more precisely with lymph node metastasis and survival. It seems that histologic subtype (see below) outperforms the tumor grade in prediction of survival, especially when combined with molecular markers [40].

\section{Histopathologic Subtype}

A paradigm shift has recently occurred in the classification of ovarian cancer. Based on morphologic and molecular features, epithelial ovarian cancer is recognized as a diverse group of tumors, amenable to a dualistic classification model. Type I tumors include low-grade serous, low-grade endometrioid, clear-cell, mucinous, and transitional cancers. Type II tumors include high-grade serous, undifferentiated, and malignant mixed mesodermal cancers. Type I tumors generally demonstrate slower growth and a lower stage at diagnosis; these tumors also tend to lack mutations involving TP53 and are generally not sensitive to 
platinum-based chemotherapy. Cell types reflect differences in underlying molecular profile and thus in sensitivity to current chemotherapy. Type II tumors display an aggressive nature and are usually advanced in stage at diagnosis; the majority of these tumors harbor TP53 mutations and are initially sensitive to platinum-based chemotherapy. Platinum sensitivity is pivotal to the treatment of advanced ovarian cancer, for which platinum-taxane combination chemotherapy remains the standard of care following surgical debulking. Tumor-cell type is shown to be the most relevant histologic prognostic factor in advanced ovarian cancer treated with platinum/paclitaxel [40].

Recent studies indicate that low-grade serous carcinoma has a significantly better prognosis than high-grade serous carcinoma [41, 42]. In advanced epithelial ovarian cancer, recent meta-analyses report that the mucinous and clear-cell subtypes are associated with worse outcome $[9,14,29,43$, 44]. Challenges to determining the prognostic importance of tumor histology include misclassification in studies that lack centralized histopathological review, heterogeneity of study populations, and small sample sizes.

\section{Obesity}

An extensive United States population-based study demonstrated an association between increased body mass index (BMI) and increased risk of death from all cancers. This study further reported a direct relationship between increasing BMI and increasing risk of mortality from ovarian cancer; women with a BMI of 35 and above had a relative risk of death of 1.51 compared to women with a normal BMI [34]. However, the authors noted that several studies have not shown such an association and the literature is inconsistent with regard to the etiologic relationship between obesity and ovarian cancer and the effect of obesity on survival [45-54]. Recently, a link has been found between the postmenopausal hormonal therapy and the effect of BMI on ovarian cancer prognosis; and given the role of the leptin in progression of various cancers, a hormonal mechanism is suggested. Indeed higher levels of leptin in ovarian cancer patients correlate with worse clinical outcome $[55,56]$.

\section{Surgical Debulking}

In accordance with a Gompertzian model of general tumor kinetics, a greater initial surgical reduction of tumor burden results in improved 5-year survival with adjuvant chemotherapeutic regimens. This understanding is based on the concept that the growth fraction of a tumor decreases with time; hence, chemotherapy destroys tumor cells based on where a tumor is on its growth curve. Smaller tumor volumes are more readily destroyed than larger volumes because the growth fraction of the smaller volume is higher and thus the fractional cell kill by a chemotherapeutic agent is also higher [57, 58]. The success of cytoreductive surgery, as reflected by the size of residual tumor, has been consistently demonstrated as one of the most influential factors of both progression-free and overall survivals $[9,13,19,21,59-61]$.
It is noteworthy that no randomized trial has been conducted to support this understanding, and almost all supportive studies are retrospective and incorporate various adjuvant chemotherapy regimens. In advanced disease, one randomized trial demonstrates that neoadjuvant chemotherapy, wherein chemotherapy is administered before attempting cytoreductive surgery, does not improve survival [62].

A recent Cochrane Database Review evaluated the effectiveness of optimal primary cytoreductive surgery for women with advanced epithelial ovarian cancer and assessed the impact of various residual tumors sizes on overall survival [63]. Analyses showed that overall survival and progressionfree survival were significantly prolonged for women who had no grossly visible residual disease following primary debulking surgery. When comparing greater than one centimeter versus less than one centimeter of residual tumor, survival estimates also favored the lower volume residual disease group. Finally, there was no significant difference in overall survival and only a borderline difference in progressionfree survival when residual disease of greater than two centimeters and less than two centimeters were compared. The authors concluded that complete cytoreduction to no grossly visible disease should be the goal of primary surgery for advanced-stage epithelial ovarian cancer but noted that the retrospective nature of the data precluded a definitive understanding of whether the surgical intervention or patient-related and disease-related factors were responsible for improved survival [63]. Interestingly, a study by the Gynecologic Oncology Group found that women who presented with large volume disease and were optimally cytoreduced to less than 1 centimeter residual tumor had inferior survival compared to women who initially presented with disease of one centimeter or less, which raises the possibility of biologic factors related to disease bulk being as important as the degree of achievable surgical cytoreduction [64].

Indeed, it has recently been shown that innate tumor features determine cytoreductibility of women with ovarian carcinoma; preoperative CA-125 and P53 can determine if the tumor would be cytoreductible or not. According to a multivariate analysis, patients with tumors that strongly express p53 are significantly less likely to achieve complete cytoreduction than the patients whose tumors had mild or moderate p53 expression [65].

Surgeon subspecialization also impacts the success of initial cytoreductive surgery and survival. Patients with early and advanced-stage disease are both more likely to receive optimal cytoreduction and more likely to experience improved median and overall survivals when operated on by a gynecologic oncologist [66].

The success of neoadjuvant chemotherapy is also an important prognostic factor for patients with ovarian cancer who are not candidates for primary surgical debulking due to the extent of their disease. Unfortunately, some of these patients are refractory to initial treatment and therefore incur a poor prognosis. A recent retrospective study was published that evaluated actionable targets in patients who received neoadjuvant chemotherapy prior to surgical debulking. Of note, hepatocyte growth factor (HGF) and its receptor cMET were enhanced in those with aggressive ovarian cancer 
and were associated with refractory disease [67]. The HGF/cMET is a known growth-factor signaling pathway. The development of novel agents to target the HGF/c-MET pathway would allow for a more tailored approach for these patients with refractory disease [67].

\section{Long-Term Prognostic Factors in Recurrent Ovarian Cancer}

Prognostic factors in recurrent ovarian cancer slightly differ from the primary tumors of the same type. Several studies using multivariate analysis with age, histology, grade, and residual tumor after first-look surgery have demonstrated that residual disease after initial surgery is the main factor significantly related to survival in patients treated with second look laparotomy. Accordingly, cytoreduction at second look laparoscopy will benefit survival only if microscopic residuals remained and this effect is only seen in those patients who start with less than $1 \mathrm{~cm}$ of disease. Also tumor grade was associated with survival in recurrent ovarian cancer [68-72]. In recurrent ovarian cancer, patients with the nonmucinous/clear-cell type, including serous, endometrioid, and other types, have significantly more favorable outcome compared with patients with the mucinous/clear-cell histological type [73].

Additionally, several molecular studies have shown differential expression of few genes in long-term survivors. In this regard, the MAL (myelin and lymphocyte protein) gene, implicated in conferring resistance to cancer therapy, is differentially upregulated in short-term survivors (threefold increase compared with long-term survivors and 29-fold increase compared with early-stage patients) [74]. Similarly, three other genes, namely, CYP4B1, CEPT1, and CHMP4A are differentially regulated in patients who suffer from recurrent ovarian cancer disease [75].

\section{Hereditary Ovarian Cancer}

Approximately $5 \%$ to $10 \%$ of epithelial ovarian cancers are associated with an inherited genetic predisposition, of which there are two recognized syndromes, inherited in an autosomal dominant pattern: the breast and ovarian cancer syndrome, linked to mutations in the BRCA1 and BRCA2 genes and accounting for approximately $90 \%$ of hereditary ovarian cancer cases, and the hereditary nonpolyposis colorectal cancer syndrome, accounting for approximately $5 \%$ of hereditary ovarian cancer cases $[76,77]$. The BRCA genes do not demonstrate equal penetrance and the lifetime risk of developing epithelial ovarian cancer for women with a BRCA1 or BRCA2 mutation is up to $66 \%$ and $27 \%$, respectively. Notably, women of Ashkenazi Jewish decent have an approximately $2 \%$ carrier rate of a BRCA germ line mutation, and a BRCA mutation is found in up to $62 \%$ of ovarian cancers in this population [78, 79]. Although attempts to identify the effects of BRCA mutations on ovarian cancer survival have yielded mixed results, most studies demonstrate a survival benefit for ovarian cancer patients with BRCA mutations compared to their sporadic counterparts [80, 81].
The frequency of poorly differentiated carcinoma is higher in BRCA related ovarian cancer compared to sporadic cases [80-83]; BRCA mutations have also been associated with a higher rate of cellular proliferation [84]. Poorer differentiation due to accelerated proliferation seems to convey a worse prognosis. Indeed reduced expression of BRCA1 is a common occurrence in advanced ovarian cancer [85].

Several mechanisms can account for this seemingly paradoxical observation that harboring BRCA1 mutations leads to a better prognosis. Ovarian cancers associated with BRCA mutations, particularly BRCA1, present at a younger age compared to sporadic cases $[80,81]$. While younger age at diagnosis is a well-established favorable prognostic factor, BRCA carrier status remains an independently significant prognostic factor after adjusting for the effects of age. Studies have established that the BRCA1 and BRCA2 genes are significantly involved in DNA damage recognition and homologous repair (HR) [86, 87]. Higher rate of cell proliferation and poor differentiation, in conjunction with impaired HR due to mutated BRCA genes, may contribute to an improved response to the DNA-damaging effects of platinum-based chemotherapy and probably a better prognosis [88-91]. This effect is similar to the setting of impaired HR induced by poly(ADP-ribose) polymerase (PARP) inhibitors which enhance the cytotoxic effect of DNA-damaging chemotherapy $[92,93]$.

\section{Immunological Factors}

In the case of ovarian cancer, the protective role of the immune system has been demonstrated as a survival benefit conferred by tumor infiltrating lymphocytes $[94,95]$. This benefit is more pronounced among patients with higher $\mathrm{CD}^{+}$cell counts, as well as those with higher $\mathrm{CD} 8^{+}$to $\mathrm{CD} 4^{+}$ cell ratios $[95,96]$. Notably, only T-cell infiltration in tumor islets demonstrates a survival benefit, while the presence of T-cells in stroma does not suggest a benefit. Additionally, the presence of $\mathrm{CD} 4^{+} \mathrm{CD} 25^{+}$T-cells, known as regulatory T-cells-a subset of T-cells with potent immunosuppressor activity, may predict poor clinical outcome $[95,96]$.

Likewise, overexpression of certain human leukocyte antigens (HLAs) and underexpression of other HLAs have been implicated in the interaction of the host immune response and ovarian cancer cells. Overexpression of human leukocyte antigen-G (HLA-G) may serve as one of the mechanisms by which ovarian cancer cells evade immune surveillance $[97,98]$. HLA-G is a nonclassical major histocompatibility complex class I molecule, and there is substantial experimental evidence to support its role in suppressing the immune response. On the other hand, underexpression of HLA class I molecules results in diminished recognition of tumor cells by cytotoxic $\mathrm{CD}^{+}$T-cells. Although this downregulation of HLA class I has been correlated with higher tumor stage [99], there is inconsistent evidence that it has independent prognostic significance in ovarian cancer $[99,100]$. 
TABLE 1: A summary of predictive and prognostic protein biomarkers in epithelial ovarian cancer.

\begin{tabular}{|c|c|}
\hline \multirow{2}{*}{$\begin{array}{l}\text { Oncogenes and tumor } \\
\text { suppressors }\end{array}$} & Tumor suppressor p53 \\
\hline & WT1 \\
\hline \multirow{3}{*}{ Proliferation markers } & Ki67 \\
\hline & Proliferation cell nuclear antigen or PCNA \\
\hline & Topoisomerases: topo II \\
\hline \multirow{2}{*}{ Cell cycle regulators } & Cyclins (E, D1, D3, and A) \\
\hline & Cyclin inhibitors: p21, p27, p57, and p16 \\
\hline \multirow{11}{*}{ Apoptosis } & Extrinsic apoptotic pathway \\
\hline & TRAIL and receptors \\
\hline & TRAIL-R1/DR-4 and TRAIL-R2/DR-5 \\
\hline & TRAIL-R3/DcR1, TRAIL-R4/Dcr2, and TRAIL-R5 \\
\hline & Fas and Fas-L \\
\hline & Intrinsic apoptotic pathway \\
\hline & The Bcl-2 family members (Bax and Bcl-2) \\
\hline & Caspases \\
\hline & Initiator: caspase- 8 \\
\hline & Effector: caspase-3/CPP32 \\
\hline & Inhibitor of apoptosis (IAPs) including Survivin and X-linked IAP (XIAP) \\
\hline \multicolumn{2}{|c|}{ DNA repair enzymes: BRCA1 and BRCA2, PARP-1, and ERCC1 } \\
\hline \multirow{2}{*}{$\begin{array}{l}\text { Markers of } \\
\text { angiogenesis }\end{array}$} & Markers of microvascular density (CD31 and CD34) \\
\hline & $\begin{array}{l}\text { Markers of proteins involved in angiogenesis (VEGF, HIF, COX-2, and MMPs especially MMP-9 and } \\
\text { MMP-7) }\end{array}$ \\
\hline $\begin{array}{l}\text { Immunological } \\
\text { factors }\end{array}$ & $\begin{array}{l}\text { T-cells, inhibitory T-Regs cells, cytokines, and costimulatory or inhibitory molecules expressed by } \\
\text { immune cells or tumor cells }\end{array}$ \\
\hline \multirow{5}{*}{$\begin{array}{l}\text { Tyrosine kinase } \\
\text { receptors (TKR) }\end{array}$} & Epidermal growth factor receptor (EGFR), Her-2, ErbB3, and ErbB4 \\
\hline & Ephrin B receptors and other TKR \\
\hline & EphB4 \\
\hline & Hepatocyte growth factor receptor (Hgf/Met) and its ligand, c-Met \\
\hline & The signaling pathway of tyrosine kinase receptors: Erk, PI3K, Akt, and NF- $\kappa \mathrm{BI}$ \\
\hline
\end{tabular}

\section{Molecular Profiling}

The most frequently studied putative molecular biological prognostic factors in ovarian cancer are the tumor suppressor protein 53 (p53), the oncogenes epidermal growth factor receptor (EGFR) and human epidermal growth factor receptor 2 (HER-2/neu). Results of a recent meta-analysis show that p53, EGFR, and HER-2/neu immunostainings do not have a strong direct relationship with survival, although likely their respective pathways do influence patient prognosis [101].

Additional biomarkers have also been previously identified as potential targets to identify prognosis and appropriate adjuvant chemotherapy after surgical debulking; however, the results have been inconclusive. It seems that, due to these conflicting results, meta-analysis studies are very helpful. For example, a recent meta-analysis evaluated Bcl-2, EGFR, GST, LRP, p16, p21, P-pg, and TNF- $\alpha$ and their relation to patient survival and response to treatment. Both EGFR and P-pg enhancements were prognostic factors for poor overall survival (OS) and poor progression free survival (PFS). Of additional utility, LRP, P-pg, and TNF were shown to be possible targets that are associated with response to platinumbased chemotherapy [102]. Table 1 shows a summary of predictive and prognostic protein biomarkers implicated in epithelial ovarian cancer [103].

The application of novel approaches based on genomic and proteomic studies has made it possible to obtain a vast amount of data about the molecular platform on which tumors arise and grow. A better understanding of these mechanisms could prove to be invaluable in sketching a roadmap towards the individualization of cancer treatment, with the ultimate goal of developing the least toxic treatment regimens to improve survival. Recently, The Cancer Genome Atlas (TCGA) project completed the first comprehensive genomic analysis of high grade serous ovarian cancer (HGS-OvCa), which accounts for the most ovarian cancer patient deaths. Using approximately 1500 intrinsically variable genes, The Cancer Genome Atlas Research Network found four clusters using unsupervised nonnegative matrix factorization that 
were termed "Differentiated," "Immunoreactive," "Mesechymal," and "Proliferative." These four clusters were not different regarding survival [104].

Nevertheless, in accordance with previous studies, TCGA found that almost all HGS-OvCa tumors are characterized by mutations in the tumor suppressor gene TP53 [105107]. Interestingly, a study derived from TCGA discovered that HGS-OvCa patients without mutated TP53 are more chemoresistant and have poorer survival compared to patients with mutated TP53 [108]. TCGA additionally found HGS-OvCa displays statistically recurrent somatic mutations in nine genes, with over $20 \%$ of HGS-OvCa tumors harboring a BRCA1 or BRCA2 mutation. As previously discussed, defective homologous recombination (HR) due to BRCA mutations may render cancer cells more sensitive to PARP inhibitors. TCGA discovered genomic alterations involving other HR genes and estimated that approximately half of all HGS-OvCa tumors may harbor HR defects, which could have implications for trials of PARP inhibitors. The project also identified twenty-two amplified, overexpressed genes that are potential targets for therapeutic inhibitors. Another recent study integrated TCGA data and validated three genes (CYP4B1, CEPT1, and CHMP4A) in HGS-OvCa from patients likely to be cured by initial cytoreductive surgery and adjuvant chemotherapy; the precise role of these genes in ovarian cancer pathophysiology remains to be elucidated [109]. While the mutational spectrum delineated by TCGA is distinct for HGS-OvCa, the findings indicate that subtypestratified care and targeted therapies may lead to future improvements in ovarian cancer treatment. In a similar approach by OVCAD consortium, by applying a gene signature comprised of 112 genes, it was shown that, in advancedstage serous ovarian cancer, two approximately equally large molecular subtypes exist, independent of classical clinocopathological parameters and presented with highly different whole-genome expression profiles and a markedly different overall survival [110]. By making the transition from the gene as the unit of phenotypic affiliation to the molecular network as the unit of analysis, both the survival can be better predicted and also the treatment can be better stratified. Thus, instead of using individual genes, the network unit can be considered as a biomarker; for example, PDGF network (18 genes) is shown in several studies to outperform the 193-gene signature in the TCGA database [111].

Furthermore, the development of novel agents based on actionable targets is dependent on their reproducibility. It has been reported that pathways and pathway-based genes are more reliable biomarkers compared to single gene markers as they are more stable and are better at identifying the subtype of cancer across data sets [112]. Additionally, utilizing multidimensional biomarkers has greater predictive value for aggressive disease and therefore greater clinical application than employing single dimension evaluation [113]. One such pathway that has been identified in epithelial cancers, including ovarian, is the BMI1 pathway, which is associated with pluripotent potential of a cell. This has been shown to reduce response to chemotherapy, decrease time to disease progression, and increase the risk for metastatic disease. The ability to target this pathway and individualize treatment for individuals with this biomarker could greatly improve survival [114].

As a sight for the future, in order to standardize diagnosis and prognosis regimens, we see that there is a strong relation between future improvements and canonical biomarkers. Using well-recognized quantitative features to encode cancer and other phenotypes based on canonical biomarkers will connect different laboratories and give such ability to record marker-based data interchangeably among them. As a result, this relation, outcomes, and information will incorporate genomic cancer information as a matter of course, augmenting current cancer classification protocols and specifying the treatments protocols [112].

This review covered various demographic, clinical, and molecular factors that influence the survival of patients with ovarian cancer. There is a concerted effort to better understand the basis of factors implicating the longevity with this disease at the clinical and molecular levels. Currently known factors affecting patient's outcome include age, performance status, and histopathologic subtype; however, new information regarding the patient's biomarkers is likely to generate the greatest predictor of patient outcome. Therefore, continued efforts at identifying prognosticators of survival may result in invaluable adjuncts to the treatment of ovarian cancer, with the ultimate goal of advancing patient care and survival.

\section{Conflict of Interests}

The authors declare that there is no conflict of interests regarding the publication of this paper.

\section{References}

[1] American Cancer Society, Cancer Facts \& Figures, American Cancer Society, Atlanta, Ga, USA, 2012.

[2] K. M. Jochumsen, Q. Tan, E. V. Høgdall et al., "Gene expression profiles as prognositic markers in women with ovarian cancer," International Journal of Gynecological Cancer, vol. 19, no. 7, pp. 1205-1213, 2009.

[3] J. R. McLaughlin, B. Rosen, J. Moody et al., "Long-term ovarian cancer survival associated with mutation in BRCA1 or BRCA2," Journal of the National Cancer Institute, vol. 105, pp. 141-148, 2013.

[4] J. Engel, R. Eckel, G. Schubert-Fritschle et al., "Moderate progress for ovarian cancer in the last 20 years: prolongation of survival, but no improvement in the cure rate," European Journal of Cancer, vol. 38, no. 18, pp. 2435-2445, 2002.

[5] A. Jemal, R. Siegel, E. Ward, T. Murray, J. Xu, and M. J. Thun, "Cancer statistics, 2007," CA: A Cancer Journal for Clinicians, vol. 57, no. 1, pp. 43-66, 2007.

[6] J. D. Hainsworth, W. W. Grosh, L. S. Burnett, and et al, "Advanced ovarian cancer: long-term results of treatment with intensive cisplatin-based chemotherapy of brief duration," Annals of Internal Medicine, vol. 108, pp. 165-170, 1988.

[7] J. P. Neijt, W. W. ten Bokkel Huinink, M. E. L. van der Burg et al., "Long-term survival in ovarian cancer: mature data from The Netherlands Joint Study Group for Ovarian Cancer," European Journal of Cancer, vol. 27, pp. 1367-1372, 1991. 
[8] M. Markman, J. L. Lewis, P. Saigo et al., "Impact of age on survival of patients with ovarian cancer," Gynecologic Oncology, vol. 49, no. 2, pp. 236-239, 1993.

[9] W. E. Winter III, G. L. Maxwell, C. Tian et al., "Prognostic factors for stage III epithelial ovarian cancer: a Gynecologic Oncology Group Study," Journal of Clinical Oncology, vol. 25, no. 24, pp. 3621-3627, 2007.

[10] U. Beller, B. Bigelow, E. M. Beckman, B. Brown, and R. I. Demopoulos, "Epithelial carcinoma of the ovary in the reproductive years: clinical and morphological characterization," Gynecologic Oncology, vol. 15, no. 3, pp. 422-427, 1983.

[11] D. Massi, T. Susini, L. Savino, V. Boddi, G. Amunni, and M. Colafranceschi, "Epithelial ovarian tumors in the reproductive age group: age is not an independent prognostic factor," Cancer, vol. 77, no. 6, pp. 1131-1136, 1996.

[12] M. M. Oken, R. H. Creech, D. C. Tormey, and et al, "Toxicity and response criteria of the Eastern Cooperative Oncology Group," The American Journal of Clinical Oncology, vol. 5, no. 6, pp. 649$655,1982$.

[13] J. K. Chan, V. Loizzi, Y. G. Lin et al., "Stages III and IV invasive epithelial ovarian carcinoma in younger versus older women: what prognostic factors are important?" Obstetrics \& Gynecology, vol. 102, pp. 156-161, 2003.

[14] J. I. Akahira, H. Yoshikawa, Y. Shimizu et al., "Prognostic factors of stage IV epithelial ovarian cancer: a multicenter retrospective study," Gynecologic Oncology, vol. 81, no. 3, pp. 398-403, 2001.

[15] B. Gronlund, C. Høgdall, H. H. Hansen, and S. A. Engelholm, "Performance status rather than age is the key prognostic factor in second-line treatment of elderly patients with epithelial ovarian carcinoma," Cancer, vol. 94, no. 7, pp. 1961-1967, 2002.

[16] R. Siegel, D. Naishadham, and J. Ahmedin, "Cancer statistics, 20113," CA: A Cancer Journal for Clinicians, vol. 63, pp. 11-30, 2013.

[17] S. M. Eisenkop, R. L. Friedman, and H. J. Wang, "Complete cytoreductive surgery is feasible and maximizes survival in patients with advanced epithelial ovarian cancer: a prospective study," Gynecologic Oncology, vol. 69, no. 2, pp. 103-108, 1998.

[18] International Federation of Gynecology and Obstetrics (FIGO) Cancer Committee, "Staging announcement," Gynecologic Oncology, vol. 25, pp. 383-385, 1986.

[19] J. L. Benedet, H. Bender, H. Jones III et al., "FIGO staging classifications and clinical practice guidelines in the management of gynecologic cancers. FIGO Committee on Gynecologic Oncology," International Journal of Gynecology \& Obstetrics, vol. 70, no. 2, pp. 209-262, 2000.

[20] R. E. Bristow, R. S. Tomacruz, D. B. Armstrong, E. L. Trimble, and F. J. Montz, "Survival effect of maximal cytoreductive surgery for advanced ovarian carcinoma during the platinum era: a meta-analysis," Journal of Clinical Oncology, vol. 20, no. 5, pp. 1248-1259, 2002.

[21] W. J. Hoskins, B. N. Bundy, and J. T. Thigpen, “The influence of cytoreductive surgery on recurrence-free interval and survival in small volume stage III epithelial ovarian cancer: a Gynecologic Oncology Group study," Gynecologic Oncology, vol. 47, pp. 167-171, 1992.

[22] R. C. Young, L. A. Walton, S. S. Ellenberg et al., "Adjuvant therapy in stage I and stage II epithelial ovarian cancer: results of two prospective randomized trials," The New England Journal of Medicine, vol. 322, no. 15, pp. 1021-1027, 1990.

[23] R. Eitan, D. A. Levine, and N. Abu-Rustum, "The clinical significance of malignant pleural effusions in patients with optimally debulked ovarian carcinoma," Cancer, vol. 103, no. 7, pp. 1397-1401, 2005.

[24] N. Colombo, D. Guthrie, S. Chiari, and et al, "International Collaborative Ovarian Neoplasm (ICON) collaborators. International Collaborative Ovarian Neoplasm trial 1: a randomized trial of adjuvant chemotherapy in women with early-stage ovarian cancer," Journal of the National Cancer Institute, vol. 95, no. 2, pp. 125-132, 2003.

[25] J. B. Trimbos, M. Parmar, I. Vergote et al., "International Collaborative Ovarian Neoplasm trial 1 and Adjuvant ChemoTherapy in Ovarian Neoplasm trial: two parallel randomized phase III trials of adjuvant chemotherapy in patients with early-stage ovarian carcinoma," Journal of the National Cancer Institute, vol. 95, no. 2, pp. 105-112, 2003.

[26] J. B. Trimbos, I. Vergote, G. Bolis et al., "ORTC-ACTION collaborators. European Organisation for Research and Treatment of Cancer-Adjuvant ChemoTherapy in Ovarian Neoplasm. Impact of adjuvant chemotherapy and surgical staging in earlystage ovarian carcinoma: European Organisation for Research and Treatment of Cancer-Adjuvant ChemoTherapy in Ovarian Neoplasm trial," Journal of the National Cancer Institute, vol. 95, no. 2, pp. 113-125, 2003.

[27] R. C. Young, "Early-stage ovarian cancer: to treat or not to treat," Journal of the National Cancer Institute, vol. 95, no. 2, pp. 94-95, 2003.

[28] D. S. Chi, J. B. Liao, L. F. Leon et al., "Identification of prognostic factors in advanced epithelial ovarian carcinoma," Gynecologic Oncology, vol. 82, no. 3, pp. 532-537, 2001.

[29] G. A. Omura, M. F. Brady, H. D. Homesley et al., "Long-term follow-up and prognostic factor analysis in advanced ovarian carcinoma: the Gynecologic Oncology Group experience," Journal of Clinical Oncology, vol. 9, no. 7, pp. 1138-1150, 1991.

[30] L. E. Puls, T. Duniho, J. E. Hunter, R. Kryscio, D. Blackhurst, and H. Gallion, "The prognostic implication of ascites in advancedstage ovarian cancer," Gynecologic Oncology, vol. 61, no. 1, pp. 109-112, 1996.

[31] T. G. Clark, M. E. Stewart, D. G. Altman et al., "A prognostic model for ovarian cancer," British Journal of Cancer, vol. 85, no. 7, pp. 944-952, 2001.

[32] D. S. Chi, M. J. Palayekar, Y. Sonoda et al., "Nomogram for survival after primary surgery for bulky stage IIIC ovarian carcinoma," Gynecologic Oncology, vol. 108, pp. 191-194, 2008.

[33] R. F. Ozols, J. Garvin, J. Costa et al., "Advanced ovarian cancer: correlation of histologic grade with response to therapy and survival," Cancer, vol. 45, pp. 572-581, 1980.

[34] E. E. Calle, C. Rodriguez, K. Walker-Thurmond et al., "Overweight, obesity, and mortality from cancer in a prospectively studied cohort of U.S. adults," The New England Journal of Medicine, vol. 348, pp. 1625-1638, 2003.

[35] J. T. Rabban and D. A. Bell, "Current issues in the pathology of ovarian cancer," The Journal of Reproductive Medicine, vol. 50, pp. 467-474, 2005.

[36] S. G. Silverberg, "Histopathologic grading of ovarian carcinoma: a review and proposal," The International Journal of Gynecological Pathology, vol. 19, pp. 7-15, 2000.

[37] International Federation of Gynecology and Obstetrics, "Classification and staging of malignant tumours in the female pelvis," Acta Obstetricia et Gynecologica Scandinavica, vol. 50, no. 1, pp. $1-7,1917$.

[38] Y. Shimizu, S. Kamoi, S. Amada et al., "Toward the development of a universal grading system for ovarian epithelial carcinoma: 
testing of a proposed system in a series of 461 patients with uniform treatment and follow-up," Cancer, vol. 82, no. 5, pp. 893-901, 1998.

[39] Y. Shimizu, S. Kamoi, S. Amada et al., "Toward the development of a universal grading system for ovarian epithelial carcinoma. I. Prognostic significance of histopathologic features-problems involved in the architectural grading system," Gynecologic Oncology, vol. 70, pp. 2-12, 1998.

[40] A. Bamias, M. Sotiropoulou, F. Zagouri et al., "Prognostic evaluation of tumor type and other histopathological characteristics in advanced epithelial ovarian cancer, treated with surgery and paclitaxel/carboplatin chemotherapy: cell type is the most useful prognostic factor," European Journal of Cancer, vol. 48, no. 10, pp. 1476-1483, 2012.

[41] C. G. Hannibal, R. Vang, J. Junge, A. Kjaerbye-Thygesen, R. J. Kurman, and S. K. Kjaer, "A binary histologic grading system for ovarian serous carcinoma is an independent prognostic factor: a population-based study of 4317 women diagnosed in Denmark 1978-2006," Gynecologic Oncology, vol. 125, no. 3, pp. 655-660, 2012.

[42] J. D. Seidman, A. Yemelyanova, and J. A. Cosin, "Survival rates for international federation of gynecology and obstetrics stage III ovarian carcinoma by cell type: a study of 262 unselected patients with uniform pathologic review," International Journal of Gynecological Cancer, vol. 22, no. 3, pp. 367-371, 2012.

[43] H. J. Mackay, M. F. Brady, A. M. Oza et al., "Prognostic relevance of uncommon ovarian histology in women with stage III/IV epithelial ovarian cancer," International Journal of Gynecological Cancer, vol. 20, no. 6, pp. 945-952, 2010.

[44] V. Hess, R. A'Hern, N. Nasiri et al., "Mucinous epithelial ovarian cancer: a separate entity requiring specific treatment," Journal of Clinical Oncology, vol. 22, pp. 1040-1044, 2004.

[45] E. A. Lew and L. Garfinkel, "Variations in mortality by weight among 750,000 men and women," Journal of Chronic Diseases, vol. 32, pp. 563-576, 1979.

[46] D. M. Purdie, C. J. Bain, P. M. Webb et al., "Body size and ovarian cancer: case-control study and systematic review (Australia)," Cancer Causes Control, vol. 12, pp. 855-863, 2001.

[47] P. J. Mink, A. R. Folsom, T. A. Sellers, and L. H. Kushi, "Physical activity, waist-to-hip ratio, and other risk factors for ovarian cancer: a follow-up study of older women," Epidemiology, vol. 7, no. 1, pp. 38-45, 1996.

[48] H. Moller, A. Mellemgaard, K. Lindvig, and et al, "Obesity and cancer risk: a Danish record-linkage study," European Journal of Cancer, pp. 344-350, 1994.

[49] C. Rodriguez, E. E. Calle, D. Fakhrabadi-Shokoohi, E. J. Jacobs, and M. J. Thun, "Body mass index, height, and the risk of ovarian cancer mortality in a prospective cohort of postmenopausal women," Cancer Epidemiology, Biomarkers \& Prevention, vol. 11, no. 9, pp. 822-828, 2002.

[50] M. Zhang, X. Xie, A. H. Lee et al., "Body mass index in relation to ovarian cancer survival," Cancer Epidemiology, Biomarkers \& Prevention, vol. 14, pp. 1307-1310, 2005.

[51] L. M. Hess, R. Barakat, C. Tian et al., "Weight change during chemotherapy as a potential prognostic factor for stage III epithelial ovarian carcinoma: a Gynecologic Oncology Group study," Gynecologic Oncology, vol. 107, pp. 260-265, 2007.

[52] C. M. Olsen, A. C. Green, D. C. Whiteman, S. Sadeghi, F. Kolahdooz, and P. M. Webb, "Obesity and the risk of epithelial ovarian cancer: a systematic review and meta-analysis," European Journal of Cancer, vol. 43, no. 4, pp. 690-709, 2007.
[53] M. M. Protani, C. M. Nagle, and P. M. Webb, "Obesity and ovarian cancer survival: a systematic review and meta-analysis," Cancer Prevention Research, vol. 5, no. 7, pp. 901-910, 2012.

[54] H. S. Yang, C. Yoon, S. K. Myung, and S. M. Park, "Effect of obesity on survival of women with epithelial ovarian cancer: a systematic review and meta-analysis of observational studies," International Journal of Gynecological Cancer, vol. 21, no. 9, pp. 1525-1532, 2011.

[55] M. F. Leitzmann, C. Koebnick, K. N. Danforth et al., "Body mass index and risk of ovarian cancer," Cancer, vol. 115, no. 4, pp. 812$822,2009$.

[56] E. S. Diaz, B. Y. Karlan, and A. J. Li, "Obesity-associated adipokines correlate with survival in epithelial ovarian cancer," Gynecologic Oncology, vol. 129, no. 2, pp. 353-357, 2013.

[57] L. Norton and R. Simon, "Tumor size, sensitivity to therapy, and design of treatment schedules," Cancer Treatment Reports, vol. 61, pp. 1307-1317, 1977.

[58] J. H. Goldie and A. J. Coldman, "A mathematic model for relating the drug sensitivity of tumors to their spontaneous mutation rate," Cancer Treatment Reports, vol. 63, pp. 1727-1733, 1979.

[59] G. R. Redman Petroni Jr. and P. E. Saigo, "Prognostic factors in advanced ovarian carcinoma," Journal of Clinical Oncology, vol. 4, pp. 515-523, 1986.

[60] C. T. Griffiths, "Surgical resection of tumor bulk in the primary treatment of ovarian carcinoma," National Cancer Institute Monograph, vol. 42, pp. 101-104, 1975.

[61] A. N. Fader and P. G. Rose, "Role of surgery in ovarian carcinoma," Journal of Clinical Oncology, vol. 25, pp. 2873-2883, 2007.

[62] J. Morrison, K. Haldar, S. Kehoe, and T. A. Lawrie, "Chemotherapy versus surgery for initial treatment in advanced ovarian epithelial cancer," Cochrane Database of Systematic Reviews, vol. 8, Article ID CD005343, 2012.

[63] A. Elattar, A. Bryant, B. A. Winter-Roach, M. Hatem, and R. Naik, "Optimal primary surgical treatment for advanced epithelial ovarian cancer," Cochrane Database of Systematic Reviews, no. 8, Article ID CD007565, 2011.

[64] P. Hoskins, I. Vergote, A. Cervantes et al., "Advanced ovarian cancer: phase III randomized study of sequential cisplatintopotecan and carboplatin-paclitaxel vs carboplatin-paclitaxel," Journal of the National Cancer Institute, vol. 102, no. 20, pp. 15471556, 2010.

[65] G. H. Eltabbakh, S. L. Mount, B. Beatty et al., "Factors associated with cytoreducibility among women with ovarian carcinoma," Gynecologic Oncology, vol. 95, no. 2, pp. 377-383, 2004.

[66] K. C. Giede, K. Kieser, J. Dodge et al., "Who should operate on patients with ovarian cancer? An evidence-based review," Gynecologic Oncology, vol. 99, pp. 447-461, 2005.

[67] M. Mariani, M. McHugh, M. Petrillo et al., "HGF/c-Met axis drives cancer aggressiveness in the neo-adjuvant setting of ovarian cancer," Oncotarget, vol. 5, no. 13, pp. 4855-4867, 2014.

[68] J. Sehouli, R. Richter, E. I. Braicu et al., "Role of secondary cytoreductive surgery in ovarian cancer relapse: who will benefit? A systematic analysis of 240 consecutive patients," Journal of Surgical Oncology, vol. 102, no. 6, pp. 656-62, 2010.

[69] J. M. Classe, I. Jaffre, J. S. Frenel et al., "Prognostic factors for patients treated for a recurrent FIGO stage III ovarian cancer: a retrospective study of 108 cases," European Journal of Surgical Oncology, vol. 37, no. 11, pp. 971-977, 2011. 
[70] I. Skírnisdóttir and B. Sorbe, "Sorbe B.: Prognostic factors for surgical outcome and 447 women treated for advanced (FIGOstages III-IV) epithelial ovarian carcinoma," International Journal of Oncology, vol. 30, no. 3, pp. 727-734, 2007.

[71] M. C. Fleisch, P. Pantke, M. W. Beckmann et al., "Predictors for interdisciplinary salvage surgery for advanced or recurrent gynecologic cancers," Journal of Surgical Oncology, vol. 95, no. 6, pp. 476-484, 2007.

[72] S. C. Dowdy, C. L. Constantinou, L. C. Hartmann et al., "Longterm follow-up of women with ovarian cancer after positive second-look laparotomy," Gynecologic Oncology, vol. 91, no. 3, pp. 563-568, 2003.

[73] H. Kajiyama, K. Shibata, M. Mizuno et al., "Long-term outcome of patients with recurrent epithelial is it the same for each histological type?" International Journal of Gynecological Cancer, vol. 22, no. 3, pp. 394-399, 2012.

[74] A. Berchuck, E. S. Iversen, J. M. Lancaster et al., "Patterns of gene expression that characterize long-term survival in advanced stage serous ovarian cancers," Clinical Cancer Research, vol. 11, no. 10, pp. 3686-3696, 2005.

[75] J. N. Barlin, P. Jelinic, N. Olvera, and et al, "Validated gene targets associated with curatively treated advanced serous ovariancarcinoma," Gynecologic Oncology, vol. 128, no. 3, pp. 512-517, 2013.

[76] J. Boyd, "Molecular genetics of hereditary ovarian cancer," Oncology (Williston Park), vol. 12, pp. 399-406, 1998.

[77] H. A. Risch, J. R. McLaughlin, D. E. Cole et al., "Prevalence and penetrance of germline $B R C A 1$ and $B R C A 2$ mutations in a population series of 649 women with ovarian cancer," American Journal of Human Genetics, vol. 68, no. 3, pp. 700-710, 2001.

[78] R. Moslehi, W. Chu, B. Karlan et al., "BRCA1 and BRCA2 mutation analysis of 208 Ashkenazi Jewish women with ovarian cancer," The American Journal of Human Genetics, vol. 66, pp. 1259-1272, 2000.

[79] B. B. Roa, A. A. Boyd, K. Volcik et al., "Ashkenazi Jewish population frequencies for common mutations in BRCA1 and BRCA2," Nature Genetics, vol. 14, pp. 185-187, 1996.

[80] A. Chetrit, G. Hirsh-Yechezkel, Y. Ben-David, E. Friedman, and S. Sadetzki, "Effect of BRCA1/2 mutations on long-term survival of patients with invasive ovarian cancer: the national Israeli study of ovarian cancer," Journal of Clinical Oncology, vol. 26, no. 1, pp. 20-25, 2008.

[81] S. C. Rubin, I. Benjamin, K. Behbakht et al., "Clinical and pathological features of ovarian cancer in women with germline mutations of BRCA1," The New England Journal of Medicine, vol. 335, pp. 1413-1416, 1996.

[82] A. Koul, S. Malander, N. Loman et al., "BRCA1 and BRCA2 mutations in ovarian cancer: covariation with specific cytogenetic features," International Journal of Gynecological Cancer, vol. 10, no. 4, pp. 289-295, 2000.

[83] S. J. Ramus, L. G. Bobrow, P. D. Pharoah, and et al, "Increased frequency of TP53 mutations in BRCA1 and BRCA2 ovarian tumours," Genes, Chromosomes and Cancer, vol. 25, no. 2, pp. 91-96, 1999.

[84] D. A. Levine, M. G. Federici, V. E. Reuter et al., "Cell proliferation and apoptosis in BRCA-associated hereditary ovarian cancer," Gynecologic Oncology, vol. 85, no. 3, pp. 431-434, 2002.

[85] W. Zheng, F. Luo, J. J. Lu et al., "Reduction of BRCA1 expression in sporadic ovarian cancer," Gynecologic Oncology, vol. 76, no. 3, pp. 294-300, 2000.
[86] R. Scully, J. Chen, A. Plug et al., "Association of BRCA1 with Rad51 in mitotic and meiotic cells," Cell, vol. 88, pp. 265-275, 1997.

[87] W. Wang, "Emergence of a DNA-damage response network consisting of Fanconi anaemia and BRCA proteins," Nature Reviews Genetics, vol. 8, pp. 735-748, 2007.

[88] J. Liu, M. C. Cristea, P. Frankel et al., "Clinical characteristics and outcomes of BRCA-associated ovarian cancer: genotype and survival," Cancer Genetics, vol. 205, no. 1-2, pp. 34-41, 2012.

[89] I. Cass, R. L. Baldwin, T. Varkey et al., "Improved survival in women with BRCA-associated ovarian carcinoma," Cancer, vol. 97, pp. 2187-2195, 2003.

[90] D. A. Levine, M. G. Federici, and V. E. Reuter, "Cell proliferation and apoptosis in BRCA-associated hereditary ovarian cancer," Gynecologic Oncology, vol. 85, no. 3, pp. 431-434, 2002.

[91] D. Yang, S. Khan, Y. Sun et al., "Association of BRCA1 and BRCA2 mutations with survival, chemotherapy sensitivity, and gene mutator phenotype in patients with ovarian cancer," Journal of American Medical Association, vol. 306, no. 14, pp. 1557-1565, 2011.

[92] J. Prat, A. Ribé, and A. Gallardo, "Hereditary ovarian cancer," Human Pathology, vol. 36, no. 8, pp. 861-870, Aug 2005.

[93] D. Bell, A. Berchuck, M. Birrer et al., "Integrated genomic analyses of ovarian carcinoma," Nature, vol. 474, no. 7353, pp. 609-615, 2011.

[94] L. Deligdisch, A. J. Jacobs, and C. J. Cohen, "Histologic correlates of virulence in ovarian adenocarcinoma. II. Morphologic correlates of host response," American Journal of Obstetrics \& Gynecology, vol. 144, pp. 885-889, 1982.

[95] E. Sato, S. H. Olson, J. Ahn et al., "Intraepithelial CD8+ tumorinfiltrating lymphocytes and a high $\mathrm{CD} 8+/$ regulatory $\mathrm{T}$ cell ratio are associated with favorable prognosis in ovarian cancer," Proceedings of the National Academy of Sciences of the United States of America, vol. 102, no. 51, pp. 18538-18543, 2005.

[96] T. J. Curiel, G. Coukos, L. Zou et al., "Specific recruitment of regulatory $\mathrm{T}$ cells in ovarian carcinoma fosters immune privilege and predicts reduced survival," Nature Medicine, vol. 10, pp. 942-949, 2004.

[97] J. J. Sheu and I.-M. Shih, "Clinical and biological significance of HLA-G expression in ovarian cancer," Seminars in Cancer Biology, vol. 17, pp. 436-443, 2007.

[98] A. Lin, W. H. Yan, H. H. Xu et al., "HLA-G expression in human ovarian carcinoma counteracts NK cell function," Annals of Oncology, vol. 18, no. 11, pp. 1804-1809, 2007.

[99] M. Vitale, G. Pelusi, B. Taroni et al., "HLA class I antigen downregulation in primary ovary carcinoma lesions: association with disease stage," Clinical Cancer Research, vol. 11, pp. 67-72, 2005.

[100] P. Rolland, S. Deen, I. Scott, L. Durrant, and I. Spendlove, "Human leukocyte antigen class I antigen expression is an independent prognostic factor in ovarian cancer," Clinical Cancer Research, vol. 13, pp. 3591-3596, 2007.

[101] P. De Graeff, A. P. Crijns, and S. de Jong, "Modest effect of p53, EGFR and HER2/neu on prognosis in epithelial ovarian cancer: a meta-analysis," British Journal of Cancer, vol. 101, no. 1, pp. 149-159, 2009.

[102] L. Xu, J. Cai, Q. Yang, and et al, "Prognostic significance of several biomarkers in epithelial ovarian cancer: a meta-analysis of published studies," Journal of Cancer Research and Clinical Oncology, vol. 139, pp. 1257-1277, 2013.

[103] C. L. Page, D. G. Huntsman, D. M. Provencher, and A.-M. Mes-Masson, "Predictive and prognostic protein biomarkers in 
epithelial ovarian cancer: recommendation for future studies," Cancers, vol. 2, no. 2, pp. 913-954, 2010.

[104] Cancer Genome Atlas Research Network, "Integrated genomic analyses of ovarian carcinoma," Nature, vol. 474, no. 7353, pp. 609-615, 2011.

[105] A. A. Ahmed, D. Etemadmoghadam, J. Temple et al., "Driver mutations in TP53 are ubiquitous in high grade serous carcinoma of the ovary," Journal of Pathology, vol. 221, no. 1, pp. 4956, 2010.

[106] J. S. Ross, S. M. Ali, K. Wang et al., "Comprehensive genomic profiling of epithelial ovarian cancer by next generation sequencing-based diagnostic assay reveals new routes to targeted therapies," Gynecologic Oncology, vol. 130, no. 3, pp. 554559, 2013.

[107] R. Salani, R. J. Kurman, R. Giuntoli, and et al, "Assessment of TP53 mutation using purified tissue samples of ovarian serous carcinomas reveals a higher mutation rate than previously reported and does not correlate with drug resistance," International Journal of Gynecological Cancer, vol. 18, no. 3, pp. 487-491, 2008.

[108] K. K. Wong, D. I. Izaguirre, S. Y. Kwan et al., "Poor survival with wild-type TP53 ovarian cancer?” Gynecologic Oncology, vol. 130, no. 3, pp. 565-569, 2013.

[109] J. N. Barlin, P. Jelinic, N. Olvera et al., "Validated gene targets associated with curatively treated advanced serous ovarian carcinoma," Gynecologic Oncology, vol. 128, no. 3, pp. 512-517, 2012.

[110] D. Pils, G. Hager, D. Tong et al., "Validating the impact of a molecular subtype in ovarian cancer on outcomes: a study of the OVCAD Consortium," Cancer Science, vol. 103, no. 7, pp. 1334-1341, 2012.

[111] R. Ben-Hamo and S. Efroni, "Biomarker robustness reveals the PDGF network as driving disease outcome in ovarian cancer patients in multiple studies," BMC Systems Biology, vol. 6, article 3, 2012.

[112] S. Kim, M. Kon, and C. deLisi, "Pathway-based classification of cancer subtypes," Biology Direct, vol. 7, article 21, 2012.

[113] M. Mariani, S. He, M. McHugh et al., "Integrated multidimensional analysis is required for accurate prognostic biomarkers in colorectal cancer," PLoS ONE, vol. 9, no. 7, Article ID e101065, 2014.

[114] G. V. Glinsky, "Stemness genomics law governs clinical behavior of human cancer: implications for decision making in disease management," Journal of Clinical Oncology, vol. 26, no. 17, pp. 2846-2853, 2008. 


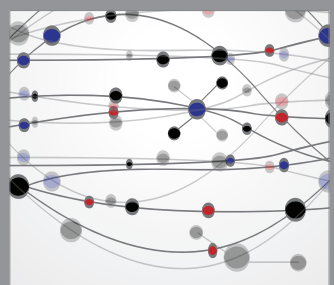

The Scientific World Journal
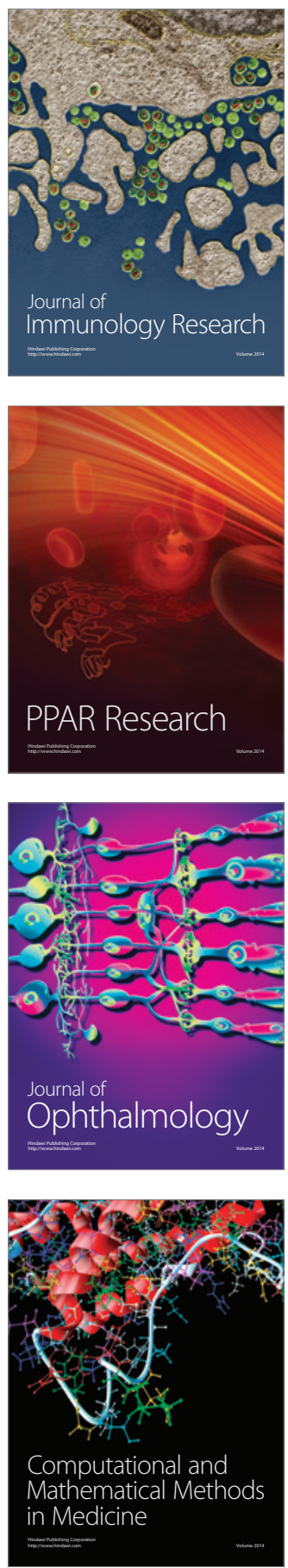

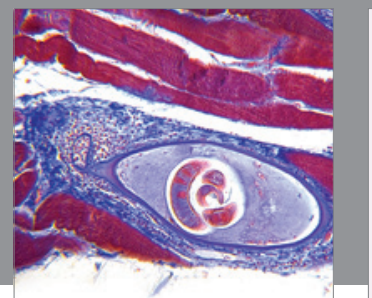

Gastroenterology

Research and Practice
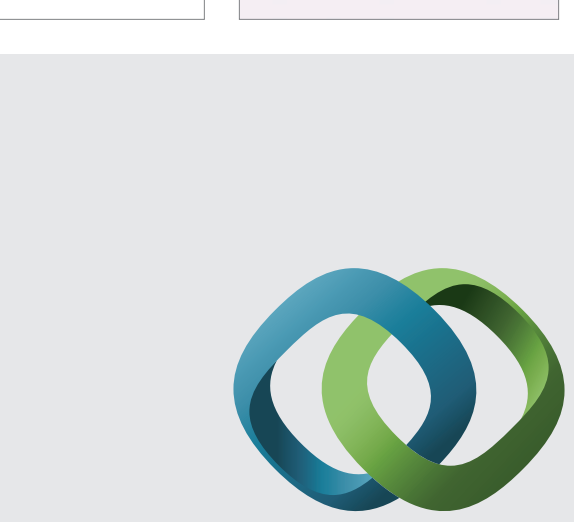

\section{Hindawi}

Submit your manuscripts at

http://www.hindawi.com
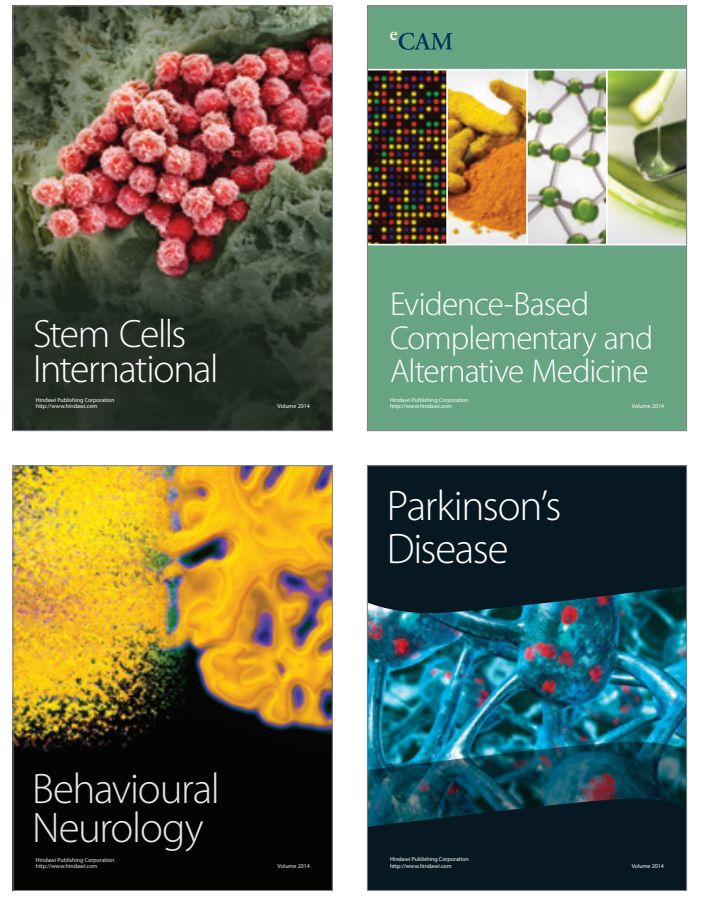
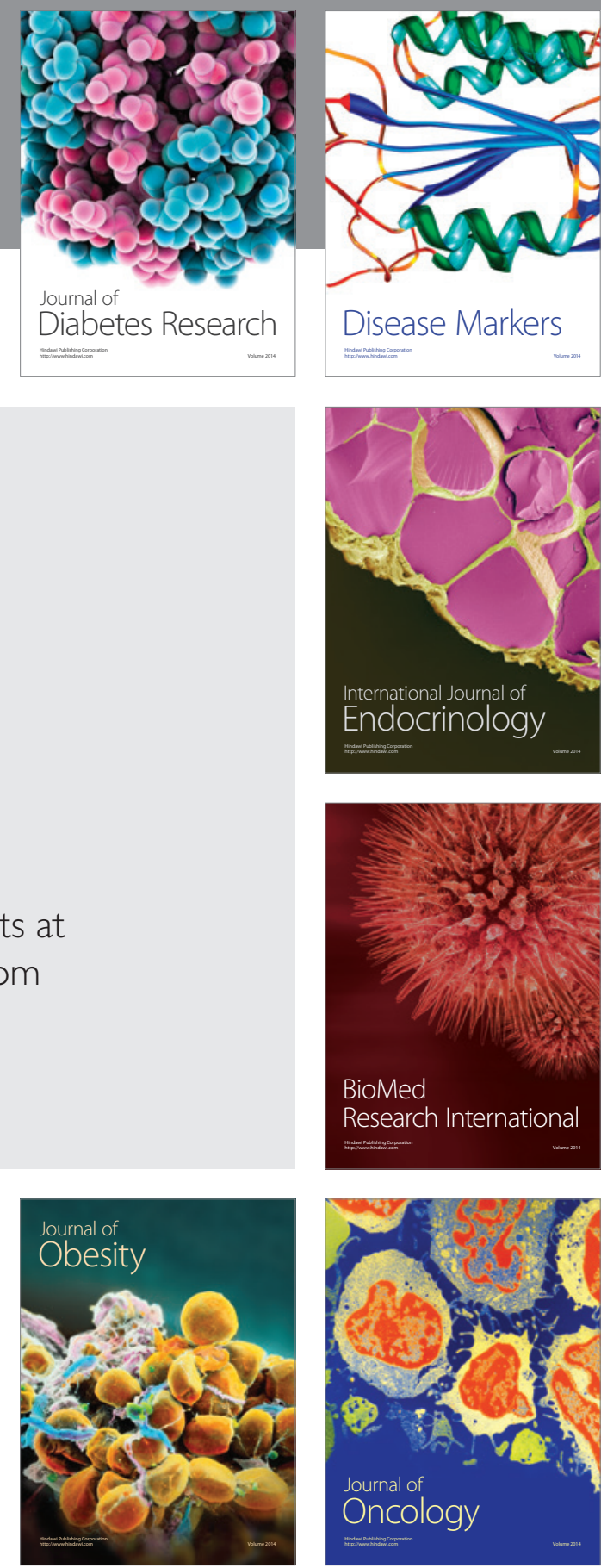

Disease Markers
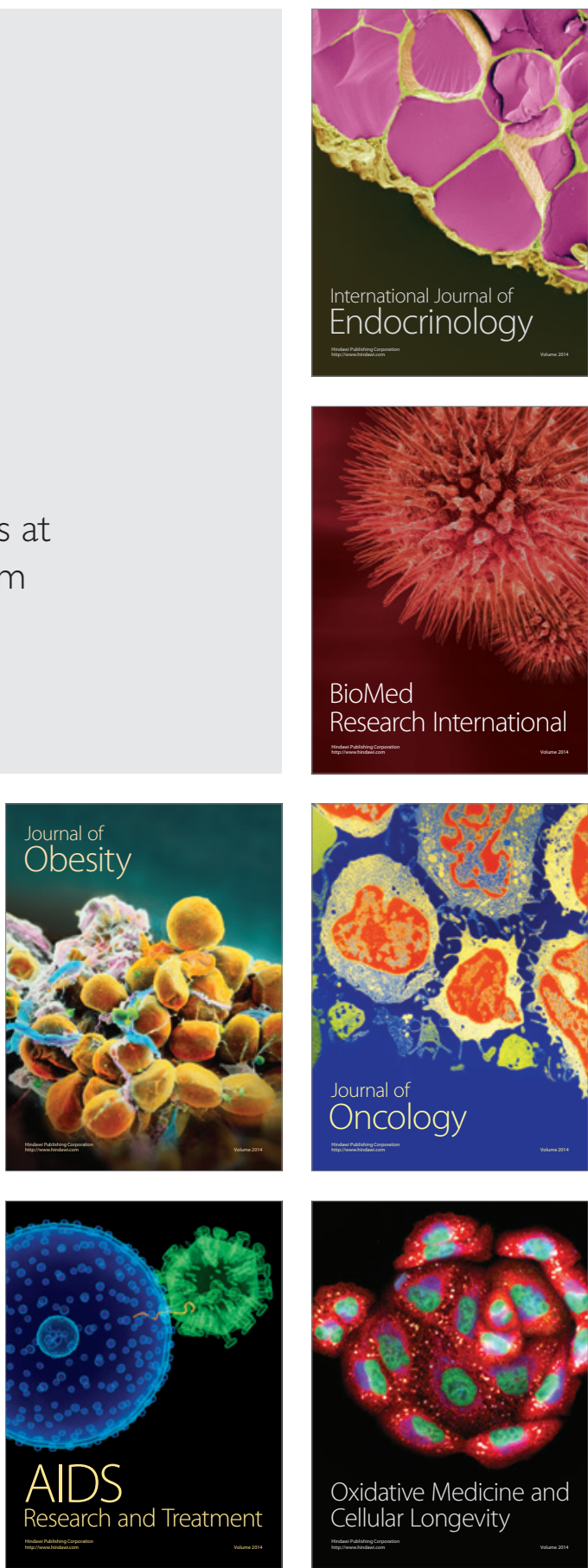